\title{
Preparation of aluminium-based quasicrystals
}

Pavel Novák, Milena Voděrová, Robin Hendrych, Tomáš Kubatík, Alena Michalcová, Dalibor Vojtěch Department of Metals and Corrosion Engineering, Institute of Chemical Technology, Prague, panovak@ vscht.cz

This paper describes the methods that are applicable for the preparation of aluminium-based quasicrystals. Application of rapid solidification of the melt, mechanical alloying and reactive sintering of metallic powders are presented. Based on the conducted experiments, mechanical alloying seems to be the most promising technology, since it enables to yield nearly pure quasicrystalline powder in $\mathrm{Al}-\mathrm{Fe}-\mathrm{Cu}$ alloy system.

Keywords: quasicrystal, aluminium, reactive sintering, mechanical alloying, rapid solidification

\section{Acknowledgement}

This research was financially supported by Czech Science Foundation, project No. P108/12/G043.

\section{References}

[1] A. MICHALCOVÁ, Kvazikrystaly aneb za co byla v roce 2011 udělena Nobelova cena, Chemické Listy, 106 (2012) 51-57.

[2] R.J. SCHAEFER, Metallurgy of quasicrystals, Scripta Metallurgica, 20 (1986) 1187-1192,.

[3] D. Pavlyuchkov, S. Balanetskyy, W. Kowalski, M. Surowiec, B. GRUSHKO, Stable decagonal quasicrystals in the Al-Fe-Cr and Al-Fe-Mn alloy systems, Journal of Alloys and Compounds, 477 (2009) L41-L44.

[4] R.W. CAHN, P. HAASEN, E.J. CRAMER, Materials Science and Technology, vol. 15: Processing of metals and alloys, VCH, Weinheim, 1991.

[5] F. ZUPANIČ, T. BONČINA, B. ŠUŠTARŠIČ, I. ANŽEL, B. MARKOLI, Microstructure of Al-Mn-Be meltspun ribbons, Materials Characterization, 59 (2008) 1245-1251.

[6] A. MICHALCOVÁ, D. VOJTĚCH, Structure of rapidly solidified aluminium alloys, Manufacturing Technology, 12 (2012) 166-169.

[7] P. NOVÁK, A. MICHALCOVÁ, J. ŠERÁK, D. VOJTĚCH, T. FABIÁN, S. RANDÁKOVÁ, F. PRŮŠA, V. KNOTEK, M. NOVÁK, Preparation of Ti-Al-Si alloys by reactive sintering, Journal of Alloys and Compounds, 470 (2009) 123-126.

[8] P. NOVÁK, D. ŠOTKA, M. NOVÁK, A. MICHALCOVÁ, J. ŠERÁK, D. VOJTĚCH, Production of NiAlmatrix composites by reactive sintering, Powder Metallurgy, 54 (2011) 308-313.

[9] P. NOVÁK, V. KNOTEK, J. ŠERÁK, A. MICHALCOVÁ, D. VOJTĚCH, Synthesis of Fe-Al-Si intermediary phases by reactive sintering, Powder Metallurgy, 54 (2009) 167-171.

[10] Š. MICHNA, P. MAJRICH, Possible ways of obtaining an aluminium alloy by non-traditional waste processing of aluminium beverage containers, Manufacturing Technology, Vol. 12, No. 13 (2012), 169-174.

[11] M. POKOVÁ, M. CIESLAR, JACQUES LACAZE, TEM Investigation of Precipitation in Al-Mn Alloys with Addition of Zr, Manufacturing Technology, Vol. 12, No. 13 (2012) 212-217.

[12] P. NOVÁK, A. MICHALCOVÁ, M. VODĚROVÁ, I. MAREK, D. VOJTĚCH, Thermal stability of Al-Cu-Fe quasicrystals prepared by SHS method, Materials Engineering, 20 (2013) 77-82. 